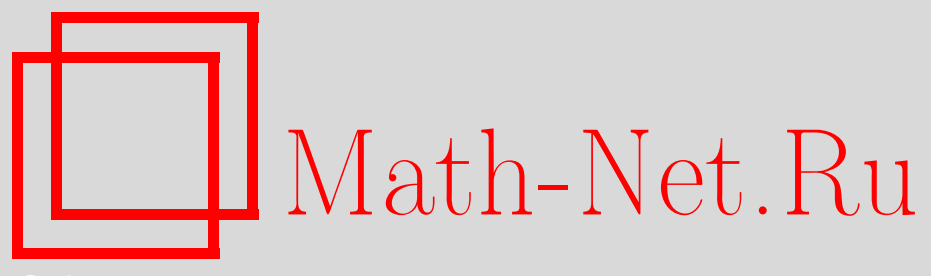

А. В. Царев, Проективные и образующие модули над кольцом псевдорациональных чисел, Матем. заметки, 2006, том 80, выпуск 3, 437-448

DOI: https://doi.org/10.4213/mzm2830

Использование Общероссийского математического портала Math-Net.Ru подразумевает, что вы прочитали и согласны с пользовательским соглашением http://www . mathnet.ru/rus/agreement

Параметры загрузки:

IP: 54.147 .182 .235

26 апреля 2023 г., 17:05:25

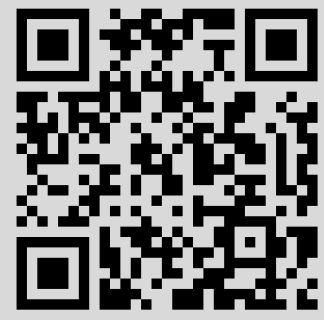




\section{ПРОЕКТИВНЫЕ И ОБРАЗУЮЩИЕ МОДУЛИ \\ НАД КОЛЬЦОМ ПСЕВДОРАЦИОНАЛЬНЫХ ЧИСЕЛ}

\section{А. В. Царев}

В работе описаны проективные, плоские и образующие модули над кольцом псевдорациональных чисел, причем для проективных модулей построена полная и независимая система инвариантов.

Библиография: 10 названий.

Кольцо псевдорациональных чисел ввели независимо друг от друга А.А. Фомин [1] и П. А. Крылов [2]. В [3] Фомин использовал конечно порожденные модули над этим кольцом для построения двойственности между категориями абелевых групп без кручения конечного ранга и факторно делимых смешанных групп с квазигомоморфизмами в качестве морфизмов. Крылов использовал модули над кольцом псевдорациональных чисел для изучения модульной структуры абелевых $s p$-групп над их кольцами эндоморфизмов (см., например, [4]).

Оба автора исследовали некоторые свойства кольца псевдорациональных чисел и модулей над ним. Так, например, и в [1] и в [2] дано описание идеалов кольца псевдорациональных чисел. Кроме того, Крыловым с соавторами доказана наследственность данного кольца, а Фоминым введен важный инвариант - псевдорациональный ранг. Отметим также, что С. В. Чегляковой в [5] описаны инъективные модули над этим кольцом. О связи модулей над кольцом псевдорациональных чисел с факторно делимыми смешанными группами и группами без кручения конечного ранга см. также [6].

Под "группой" в работе подразумевается абелева группа, записанная аддитивно, под "кольцом" - коммутативное кольцо, под "модулем" - двусторонний модуль над коммутативным кольцом; $\mathbb{Z}, \mathbb{Q}$ и $\widehat{\mathbb{Z}}_{p}$ - обозначения колец целых, рациональных и целых $p$-адических чисел соответственно или их аддитивных групп, $\mathbb{Z}(m)$ - кольцо (аддитивная группа) классов вычетов по модулю $m, P$ - множество всех простых чисел, $\mathbb{N}$ - множество всех натуральных чисел. Если $S$ - подмножество $K$-модуля $M$, то через $\langle S\rangle$ и $\langle S\rangle_{K}$ будем обозначать соответственно подгруппу и подмодуль, порожденные множеством $S$, а через $\langle S\rangle_{*}$ - сервантную оболочку множества $S$, состоящую из всех таких $r \in M$, что $n r \in\langle S\rangle$ при некотором натуральном $n$. Через $r^{*}(M)$ будем обозначать псевдорациональный ранг $R$-модуля $M$, а через $t M$ - его периодический подмодуль.

Другие используемые в работе понятия и обозначения можно найти в [7] и [8]. 


\section{1. Идеалы кольца $R$.}

ОПРЕДЕЛЕНИЕ 1 . Пусть $\chi=\left(m_{p}\right)$ - произвольная характеристика и $K_{p}=\mathbb{Z}\left(p^{m_{p}}\right)$ или $K_{p}=\widehat{\mathbb{Z}}_{p}$, соответственно при $m_{p}<\infty$ и $m_{p}=\infty$. Если $\chi$ содержит бесконечно много ненулевых $p$-компонент, то рассмотрим подкольцо $R_{\chi}$ кольца $\prod_{p \in P} K_{p}$, сервантно порожденное идеалом $\bigoplus_{p \in P} K_{p}$ и единицей кольца:

$$
R_{\chi}=\left\langle 1, \bigoplus_{p \in P} K_{p}\right\rangle_{*} .
$$

Если все $p$-компоненты характеристики $\chi$, за исключением $p_{1}, \ldots, p_{n}$, равны нулю, то определим $R_{\chi}=\mathbb{Q} \oplus K_{p_{1}} \oplus \cdots \oplus K_{p_{n}}$. Если $\chi=(\infty, \infty, \ldots)$ - характеристика группы рациональных чисел, то кольцо $R_{\chi}$ будем называть кольцом псевдорациональных чисел и обозначать просто $R$.

Для характеристики $\chi$, все $p$-компоненты которой, за исключением $p_{1}, \ldots, p_{n}$, равны нулю, определим еще кольцо $K_{\chi}=K_{p_{1}} \oplus \cdots \oplus K_{p_{n}}$.

Свойства колец $R_{\chi}$. 1 . Если характеристика $\chi$ содержит бесконечно много ненулевых компонент, то элемент $r=\left(\alpha_{p}\right) \in \prod_{p \in P} K_{p}$ принадлежит кольцу $R_{\chi}$ тогда и только тогда, когда существует такое рациональное число $|r|=m / n$, что $n \alpha_{p}=m$ почти при всех простых $p$.

2. Элемент, все $p$-компоненты которого, включая и рациональную (если такая есть), равны 1 (0), принадлежит $R_{\chi}$ и является единицей (нулем) кольца $R_{\chi}$.

3 . Обозначим через $\varepsilon_{p}$ элемент кольца $R_{\chi}$ такой, что его $p$-компонента равна 1 , а все остальные компоненты (включая и рациональную, если такая содержится) равны 0 . Тогда $\varepsilon_{p}$ - идемпотент, а множество $\varepsilon_{p} R_{\chi}$ является идеалом кольца $R_{\chi}$ и выделяется прямым слагаемым.

4. Элементы вида

(а) $\varepsilon=\varepsilon_{p_{1}}+\cdots+\varepsilon_{p_{n}}$, где $p_{1}, \ldots, p_{n}$ - различные простые числа, и элементы вида

(b) $1-\varepsilon$,

а также 1 и 0 , составляют множество всех идемпотентов кольца $R_{\chi}$.

5. Если характеристика $\chi$ содержит бесконечно много ненулевых компонент, то для элемента $r \in R_{\chi}$ обозначим через $|r|$ рациональное число, определенное в свойстве 1 , а если характеристика $\chi$ почти нулевая, то через $|r|$ обозначим рациональную компоненту элемента $r$. Тогда число $|r|$ определено однозначно и

$$
\left|r_{1} \pm r_{2}\right|=\left|r_{1}\right| \pm\left|r_{2}\right|, \quad\left|r_{1} \cdot r_{2}\right|=\left|r_{1}\right| \cdot\left|r_{2}\right|
$$

для любых $r_{1}, r_{2} \in R_{\chi}$.

6. Любой элемент $r \in R_{\chi}$ можно представить в виде $r=\varepsilon r+(1-\varepsilon)|r|$, где $\varepsilon-$ идемпотент вида (а).

7. Множество $T_{\chi}=\bigoplus_{p \in P} K_{p}$ является максимальным идеалом кольца $R_{\chi}$, причем $R_{\chi} / T_{\chi} \cong \mathbb{Q}$. Если $\chi=(\infty)$, то идеал $T_{\chi}$ будем обозначать $T$.

8. Если идеал кольца $R_{\chi}$ содержит такой элемент $r$, что $|r| \neq 0$, то он содержит идемпотент вида (b).

Рассмотрим кольцо $R_{\chi}$, построенное на характеристике $\chi=\left(m_{p}\right)$. Пусть $J-$ произвольный идеал кольца $R_{\chi}, T_{J}=T_{\chi} \cap J$ - множество всех таких $r \in J$, что $|r|=0$. Возможны два случая. 
1-й случай. $T_{J}=J$. Пусть $\varepsilon_{p} J=J_{p}$. Рассмотрим отображения $\pi_{i}: J \rightarrow J_{p_{i}}$ по закону $\pi_{i}(r)=\varepsilon_{p_{i}} r$. Для них, очевидно, выполняются условия

(a) $\pi_{i} \pi_{j}= \begin{cases}0, & \text { при } i \neq j, \\ \pi_{i}, & \text { при } i=j,\end{cases}$

(b) для любого $r \in J$

$$
r=\varepsilon r=\varepsilon_{p_{1}} r+\cdots+\varepsilon_{p_{n}} r=\pi_{1}(r)+\cdots+\pi_{n}(r) .
$$

Тогда эти отображения задают прямое разложение

$$
J=\bigoplus_{p \in P} J_{p} .
$$

Так как $J_{p}=\varepsilon_{p} J-$ идеал кольца $\varepsilon_{p} R_{\chi}=K_{p}$, то

$$
J_{p}=0 \quad \text { или } \quad J_{p}=p^{s_{p}} K_{p}, \quad s_{p}<m_{p} .
$$

Таким образом, из (1) и (2) следует, что

$$
J=\bigoplus_{p \in P_{1}} p^{s_{p}} K_{p} \cong T_{\varphi}
$$

где $P_{1}=\left\{p \in P \mid J_{p} \neq 0\right\}$, а характеристика $\varphi=\left(k_{p}\right)$ такая, что $k_{p}=m_{p}-s_{p}$ при $p \in P_{1}$ и $k_{p}=0$ при $p \notin P_{1}$.

2 -й случай. В $J$ существует такой элемент $r$, что $|r|=m / n \neq 0$. Тогда для элемента $s \in R_{\chi}$ такого, что $|s|=n / m$, справедливо $s r=t \in J$, причем $|t|=1$. Так как $u t \in J$ для любого $u \in R_{\chi}$, то в идеале $J$ есть элементы из каждого класса смежности множества $R_{\chi} / T_{\chi}$.

Очевидно, что для $r, s \in J$ равенство $|r|=|s|$ выполняется тогда и только тогда, когда $r-s \in T_{J}$. Значит, идеал $J$ состоит из подклассов $r+T_{J}$, взятых по одному из каждого класса $r+T_{\chi}$.

Так как любой класс, содержащийся в $J$, можно записать в виде $r t+T_{J}$, где $r \in R_{\chi}, t \in J$ и $|t|=1$, то идеал $J$ однозначно определяется классами $T_{J}$ и $t+T_{J}$.

Убедимся, что идеал $J$ однозначно определяется одним множеством $T_{J}$. Пусть $J_{1}$ - произвольный идеал кольца $R_{\chi}$, не содержащийся в $T_{\chi}$, такой, что $T_{J_{1}}=T_{J}$. Возьмем такие элементы $t_{1} \in J_{1}$ и $t \in J$, что $\left|t_{1}\right|=|t|=1$. Тогда $t_{1} t \in J$ и $t_{1} t \in J_{1}$. Так как $\left|t_{1} t\right|=1$, то

$$
t_{1} t+T_{J}=t_{1}+T_{J}=t+T_{J}
$$

следовательно, идеалы $J$ и $J_{1}$ совпадают, так как определяются одними и теми же классами $T_{J}$ и $t+T_{J}$.

Так как $J \not \subset T_{\chi}$, то идеал $J$ содержит такой элемент $r$, что $|r| \neq 0$; следовательно, в $J$ содержится идемпотент $1-\varepsilon$ вида (b). Тогда

$$
T_{J}=(1-\varepsilon) T_{\chi} \oplus p_{1}^{k_{1}} K_{p_{1}} \oplus \cdots \oplus p_{n}^{k_{n}} K_{p_{n}},
$$

где $\varepsilon_{p_{i}}(1-\varepsilon)=0$ для любого $i \in\{1, \ldots, n\}$. Учитывая, что $J$ однозначно определяется множеством $T_{J}$, получаем

$$
J=(1-\varepsilon) R_{\chi} \oplus p_{1}^{k_{1}} K_{p_{1}} \oplus \cdots \oplus p_{n}^{k_{n}} K_{p_{n}} .
$$

Таким образом, получили теорему. 
ТеОрема 1. Множества следующего вида, и только они, являются идеалами кольиа $R_{\chi}$ :

1) $\bigoplus_{p \in P_{1}} p^{s_{p}} K_{p}$, где $P_{1} \subseteq P$;

2) $\left(1-\varepsilon_{p_{1}}-\cdots-\varepsilon_{p_{n}}\right) R_{\chi} \oplus \bigoplus_{p \in P_{1}} p^{s_{p}} K_{p}$, где $P_{1} \subseteq\left\{p_{1}, \ldots, p_{n}\right\}$.

СЛЕДСТВИЕ 1. Любой конечно порожденный идеал кольца $R_{\chi}$ главный.

\section{2. Проективные и плоские $R$-модули.}

ОПРЕДЕЛЕНИЕ 2. Модуль $M$ называется проективным, если он удовлетворяет следующим эквивалентным условиям:

1) каждый эпиморфизм $\xi: B \rightarrow M$ расщепляется, т.е. $B=\operatorname{ker} \xi \oplus A$;

2) для любого эпиморфизма $\alpha: A \rightarrow B$ и любого гомоморфизма $\varphi: M \rightarrow B$ существует такой гомоморфизм $\psi: M \rightarrow A$, что $\varphi=\alpha \psi$;

3) модуль $M$ изоморфен прямому слагаемому свободного модуля.

Напомним, что кольцо $K$ называется наследственным, если любой его идеал является проективным модулем над $K$.

ТеОрема 2. Кольцо $R_{\chi}$ является наследственным тогда и толъко тогда, когда характеристика ұ содержит только символь 0,1 и $\infty$. В частности, кольцо псевдорациональных чисел наследственно.

ДокАЗАТЕЛЬСтво. Пусть характеристика $\chi=\left(m_{p}\right)$ удовлетворяет условиям теоремы. Идеалы вида $(1-\varepsilon) R_{\chi}$ всегда проективны над кольцом $R_{\chi}$, так как выделяются прямыми слагаемыми в $R_{\chi}$. Идеалы вида $p^{k_{p}} K_{p}, 0<k_{p}<m_{p}$, либо нулевые при $m_{p}=0$, либо равны $\mathbb{Z} / p \mathbb{Z}$ при $m_{p}=1$ и $k_{p}=0$, либо изоморфны $\widehat{\mathbb{Z}}_{p}$ при $m_{p}=\infty$; следовательно, они также проективны. Учитывая, что каждый идеал кольца $R_{\chi}$ является прямой суммой выше рассмотренных идеалов, получаем, что любой идеал кольца $R_{\chi}$ проективен.

Если характеристика $\chi$ содержит элемент $m_{p}$ такой, что $1<m_{p}<\infty$, то кольцо $R_{\chi}$ содержит идеал $p K_{p}$, который, очевидно, не проективен.

Учитывая, что проективные модули над наследственными кольцами изоморфны прямым суммам идеалов, получаем следующее утверждение.

СлеДСТВИЕ 2. Модуль $M$ проективен над колъцом псевдорациональных чисел тогда и толъко тогда, когда

$$
M \cong \bigoplus_{i \in I}\left(1-\varepsilon_{i}\right) R \oplus \bigoplus_{p \in P} \bigoplus_{\mathfrak{m}_{p}} \widehat{\mathbb{Z}}_{p}
$$

При доказательстве следующей теоремы используется тот факт, что любой подмодуль проективного модуля над наследственным кольцом проективен.

Теорема 3. Пусть $M_{1}$ и $M_{2}$ - произвольные проективные $R$-модули. Тогда если $T M_{1} \cong T M_{2}$ u $M_{1} / T M_{1} \cong M_{2} / T M_{2}$, mo $M_{1} \cong M_{2}$.

ДокАЗАтельство. Рассмотрим коммутативную диаграмму

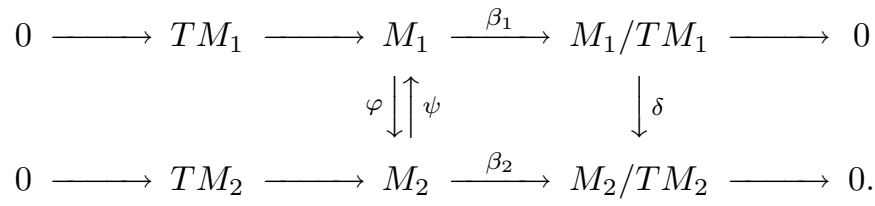


Здесь $\delta$ - изоморфизм, а гомоморфизмы $\varphi$ и $\psi$ существует в силу проективности модулей $M_{1}, M_{2}$ и определяются равенствами

$$
\beta_{2} \varphi=\delta \beta_{1}, \quad \beta_{1} \psi=\delta^{-1} \beta_{2} .
$$

Из (3) следует, что $\beta_{1}=\delta^{-1} \beta_{2} \varphi$ и $\beta_{2}=\delta \beta_{1} \psi$, откуда

$$
\begin{aligned}
& \beta_{2}=\delta\left(\delta^{-1} \beta_{2} \varphi\right) \psi=\beta_{2} \varphi \psi \Longrightarrow \beta_{2}\left(1_{M_{2}}-\varphi \psi\right)=0, \\
& \beta_{1}=\delta^{-1}\left(\delta \beta_{1} \psi\right) \varphi=\beta_{1} \psi \varphi \Longrightarrow \beta_{1}\left(1_{M_{1}}-\psi \varphi\right)=0
\end{aligned}
$$

Тогда для любых $a \in M_{1}, b \in M_{2}$

$$
\psi \varphi(a)=a+t_{1}, \quad \varphi \psi(b)=b+t_{2} \quad t_{1} \in T M_{1}, \quad t_{2} \in T M_{2} .
$$

Рассмотрим отображение $\omega=1_{M_{2}}-\varphi \psi$. Согласно (4) $\omega$ есть гомоморфизм между $R$-модулями $M_{2}$ и $T M_{2}$. По основной теореме о гомоморфизме

$$
\omega\left(M_{2}\right) \cong M_{2} / \operatorname{ker} \omega
$$

Так как $\omega\left(M_{2}\right) \subseteq T M_{2}$ и $T M_{2}$ - проективный $R$-модуль, то $\omega\left(M_{2}\right)$ также проективный $R$-модуль. А тогда из $(5)$ и проективности $\omega\left(M_{2}\right)$ следует равенство

$$
M_{2}=\omega\left(M_{2}\right) \oplus \operatorname{ker} \omega, \quad \omega\left(M_{2}\right) \subseteq T M_{2} .
$$

Обозначим $\omega\left(M_{2}\right)=T_{2}, \operatorname{ker} \omega=S_{2}$; тогда $M_{2}=T_{2} \oplus S_{2}$. Заметим, что $b \in \operatorname{ker} \omega$ тогда и только тогда, когда $\varphi \psi(b)=b$; следовательно,

$$
S_{2}=\left\{b \in M_{2} \mid \varphi \psi(b)=b\right\} .
$$

Аналогично доказывается, что $M_{1}=T_{1} \oplus S_{1}$, где

$$
S_{1}=\left\{a \in M_{1} \mid \psi \varphi(a)=a\right\}
$$

Если $a \in S_{1}$, то согласно (7) верно, что $\psi \varphi(a)=a$. Так как $\varphi \psi(\varphi(a))=\varphi(a)$, то $\varphi(a) \in S_{2}$, и значит,

$$
a=\psi(\varphi(a)) \in \psi\left(S_{2}\right)
$$

следовательно, $S_{1} \subseteq \psi\left(S_{2}\right)$. А если $c \in \psi\left(S_{2}\right)$, то $c=\psi(b)$, где $b \in S_{2}$. Тогда $\varphi(c)=\varphi \psi(b)=b$, откуда $\psi \varphi(c)=\psi(b)=c$, т.е. $c \in S_{1}$ и $\psi\left(S_{2}\right) \subseteq S_{1}$. Таким образом,

$$
\psi\left(S_{2}\right)=S_{1}
$$

Из (7) следует, что $R$-модули $S_{2}$ и $\psi\left(S_{2}\right)$ изоморфны, значит, в соответствии с равенством (8), изоморфны $R$-модули $S_{1}$ и $S_{2}$.

Так как $M_{2}=S_{2} \oplus T_{2}$, где $T_{2}=\omega\left(M_{2}\right) \subseteq T M_{2}$, то $T M_{2}=T S_{2} \oplus T_{2}$. Аналогично, $T M_{1}=T S_{1} \oplus T_{1}$. Учитывая, что $S_{1} \cong S_{2}$ и $T M_{1}, T M_{2}$ - прямые суммы проективных (свободных) $\widehat{\mathbb{Z}}_{p}$-модулей (по различным простым $p$ ), получаем, что $T_{1} \cong T_{2}$. Таким образом, $R$-модули $M_{1}$ и $M_{2}$ изоморфны. Теорема доказана. 
Пусть $M$ - произвольный проективный $R$-модуль. Рассмотрим его подмодуль $T M$. Он также проективен, более того, $T M=\bigoplus_{p \in P} M_{p}$, где $M_{p}-$ свободный $\widehat{\mathbb{Z}}_{p^{-}}$ модуль. Каждое слагаемое $M_{p}$ с точностью до изоморфизма задается кардинальным числом $\mathfrak{r}_{p}$, равным мощности множества прямых слагаемых вида $\widehat{\mathbb{Z}}_{p}$ в его свободном разложении. Далее, фактормодуль $M / T M$ является векторным пространством над полем рациональных чисел; следовательно, задается величиной $\operatorname{dim}_{\mathbb{Q}} M / T M$, называемой псевдорациональным рангом $R$-модуля $M$ (далее его будем обозначать $\left.r^{*}(M)\right)$.

Из всего выше сказанного и теоремы 3 следует, что псевдорационалъный ранг и множество кардинальных чисел $\left\{\mathfrak{r}_{p}\right\}_{p \in P}$ составляют полную и независимую систему инвариантов проективного $R$-модуля $M$.

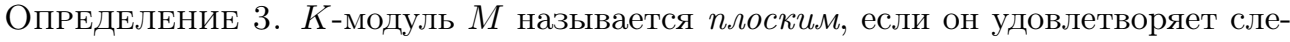
дующим эквивалентным условиям:

1) каждый мономорфизм $\alpha: A \rightarrow B$ индуцирует мономорфизм

$$
\alpha \otimes 1_{M}: A \otimes_{K} M \rightarrow B \otimes_{K} M
$$

$2)$ для любого конечно порожденного идеала $I$ кольца $K$ канонический эпиморфизм $\beta: I \otimes_{K} M \rightarrow I M$ по закону $\beta(i \otimes m)=$ im является изоморфизмом;

$3)$ модуль $M$ является прямым пределом проективных $K$-модулей.

Из теоремы 1 следует, что конечно порожденные идеалы кольца псевдорациональных чисел имеют вид

$$
I_{1}=\bigoplus_{p \in P_{1}} p^{s_{p}} \widehat{\mathbb{Z}}_{p} \quad \text { или } \quad I_{2}=(1-\varepsilon) R \oplus \bigoplus_{p \in P_{1}} p^{s_{p}} \widehat{\mathbb{Z}}_{p}=(1-\varepsilon) R \oplus I_{1},
$$

где $P_{1}$ - произвольное конечное множество простых чисел и $(1-\varepsilon) I_{1}=0$. Тогда

$$
I_{1} \otimes_{R} M \cong \bigoplus_{p \in P_{1}}\left[p^{s_{p}} \widehat{\mathbb{Z}}_{p} \otimes_{R} M\right], \quad I_{2} \otimes_{R} M \cong\left[(1-\varepsilon) R \otimes_{R} M\right] \oplus\left[I_{1} \otimes_{R} M\right] .
$$

Так как модуль $(1-\varepsilon) R \otimes_{R} M$ канонически изоморфен модулю $(1-\varepsilon) M$, из прямого разложения модуля $I_{1} \otimes_{R} M$ и условия 2) определения плоского модуля следует, что $M$ является плоским $R$-модулем тогда и только тогда, когда $M_{p}=\varepsilon_{p} M-$ плоский $\widehat{\mathbb{Z}}_{p}$-модуль при любом простом $р$.

Так как $\widehat{\mathbb{Z}}_{p}$ - коммутативная наследственная область целостности, то $p$-адический модуль $M_{p}$ является плоским в том и только том случае, когда $M_{p}$ есть модуль без кручения, что равносильно тому, что в $M_{p}$ нет элементов порядка $p$.

Таким образом, из всего выше сказанного следует

ТЕОРема 4. Модуль над кольцом псевдорачиональных чисел является плоским тогда и толъко тогда, когда в нем нет элементов конечного порядка, отличных от нуля.

Вернемся к вопросу о проективности для случая конечно порожденных $R$-модулей. Для начала докажем две вспомогательные леммы.

ЛЕмма 1. Если $L-$ подмодулъ $R$-модуля $M, \operatorname{mo} r^{*}(M)=r^{*}(M / L)+r^{*}(L)$. 
ДокАЗАТЕЛЬСтво. Так как $T(M / L)=T M / T L$, то

$$
(M / L) / T(M / L)=(M / L) /(T M / T L) \cong(M / T M) /(L / T L) .
$$

Следовательно, $r^{*}(M)=r^{*}(M / L)+r^{*}(L)$.

Далее, пусть $X=\left\{x_{1}, \ldots, x_{n}\right\}-$ произвольная система элементов $R$-модуля $M$. Рассмотрим множество

$$
\Delta M_{X}=\left\{\left(r_{1}, \ldots, r_{n}\right) \in \mathbb{R}^{n} \mid r_{1} x_{1}+\cdots+r_{n} x_{n}=0\right\}
$$

которое, очевидно, является $R$-модулем. Если $X$ - система образующих в $M$, то $\Delta M_{X}$ будем называть модулем псевдорачиональных отношений $R$-модуля $M$.

Лемма 2. Если $X=\left\{x_{1}, \ldots, x_{n}\right\}$ - система образующих $R$-модуля $M$, mо

$$
n=r^{*}(M)+r^{*}\left(\Delta M_{X}\right) .
$$

ДокАЗАтельство. Рассмотрим отображение $\varphi: \mathbb{R}^{n} \rightarrow M$ по закону

$$
\varphi\left(r_{1}, \ldots, r_{n}\right)=r_{1} x_{1}+\cdots+r_{n} x_{n}
$$

Нетрудно убедиться, что $\varphi$ - эпиморфизм. Заметим, что

$$
\left(r_{1}, \ldots, r_{n}\right) \in \operatorname{ker} \varphi \Longleftrightarrow r_{1} x_{1}+\cdots+r_{n} x_{n}=0,
$$

т.е. $\operatorname{ker} \varphi=\Delta M_{X} ;$ следовательно, $M \cong \mathbb{R}^{n} / \Delta M_{X}$ и $n=r^{*}(M)+r^{*}\left(\Delta M_{X}\right)$.

ТЕОрема 5. Конечно порожденный $R$-модуль раскладывается в прямую сумму проективного и конечного модуля тогда и только тогда, когда у него есть конечно порожденный модуль псевдорациональных отношений.

ДокАзАТЕЛЬСтво. Пусть конечно порожденный $R$-модуль $M$ раскладывается в прямую сумму проективного и конечного модуля. Тогда

$$
M \cong\left(1-\varepsilon_{1}\right) R \oplus \cdots \oplus\left(1-\varepsilon_{n}\right) R \oplus K_{\chi_{1}} \oplus \cdots \oplus K_{\chi_{m}}
$$

Рассмотрим множество $X$, в которое входит по одному образующему элементу из каждого слагаемого разложения (9). Нетрудно видеть, что тогда

$$
\Delta M_{X} \cong \varepsilon_{1} R \oplus \cdots \oplus \varepsilon_{n} R \oplus\left(1-\varepsilon_{n+1}\right) R \oplus \cdots \oplus\left(1-\varepsilon_{n+m}\right) R,
$$

т.е. $M$ имеет конечно порожденный модуль псевдорациональных отношений.

Обратно, пусть $M$ имеет конечно порожденный модуль псевдорациональных отношений $\Delta M_{X}$, построенный на множестве образующих $X=\left\{x_{1}, \ldots, x_{n}\right\}$.

Рассмотрим множество

$$
\Delta^{*} M_{X}=\left\{\left(r_{1}, \ldots, r_{n}\right) \in \mathbb{R}^{n} \mid r_{1} x_{1}+\cdots+r_{n} x_{n} \in t M\right\}
$$

где $t M$ - подмодуль $R$-модуля $M$, состоящий из всех элементов конечного порядка. Очевидно, $\Delta^{*} M_{X}$ является модулем псевдорациональных отношений $R$-модуля $M / t M$, построенным на множестве образующих $\bar{X}=\left\{x_{1}+t M, \ldots, x_{n}+t M\right\}$.

Так как $t M \subseteq T M$, то $r^{*}(t M)=0$; следовательно, $r^{*}(M / t M)=r^{*}(M)$. По лемме 2 отсюда получаем, что $r^{*}\left(\Delta^{*} M_{X}\right)=r^{*}\left(\Delta M_{X}\right)$. 
Поскольку кольцо $R$ наследственное, то из $\Delta^{*} M_{X} \subseteq \mathbb{R}^{n}$ и $\Delta M_{X} \subseteq \mathbb{R}^{n}$ получаем, что $R$-модули $\Delta^{*} M_{X}$ и $\Delta M_{X}$ проективные. Тогда, учитывая, что $r^{*}\left(\Delta^{*} M_{X}\right)=$ $r^{*}\left(\Delta M_{X}\right)$, имеют место разложения

$$
\Delta^{*} M_{X}=P_{1} \oplus S_{1} \quad \text { и } \quad \Delta M_{X}=P_{2} \oplus S_{2},
$$

где $P_{1} \cong P_{2} \cong(1-\varepsilon) R^{k}$, а модули $S_{1}$ и $S_{2}$ имеют псевдорациональный ранг 0, причем $S_{2}$ конечно порожден.

Предположим, что $R$-модуль $\Delta^{*} M_{X}$ не является конечно порожденным. Тогда $S_{1}$ содержит подмодуль $L$ вида $\bigoplus_{p \in P_{1}} \widehat{\mathbb{Z}}_{p}$, где $P_{1}$ - бесконечное множество простых чисел. Пусть $\left\{\alpha_{i}=\left(\alpha_{i 1}, \ldots, \alpha_{i n}\right) \mid i \in \mathbb{N}\right\}$ - независимое над $R$ множество порождающих модуля $L$. Так как

$$
\alpha_{i 1} x_{1}+\cdots+\alpha_{i n} x_{n} \in t M \Longleftrightarrow m_{i} \alpha_{i 1} x_{1}+\cdots+m_{i} \alpha_{i n} x_{n}=0
$$

при некотором натуральном $m_{i}$, то $S_{1}$ также содержит подмодуль вида $\bigoplus_{p \in P_{1}} \widehat{\mathbb{Z}}_{p}$, порожденный множеством $\left\{m_{i} \alpha_{i} \mid i \in \mathbb{N}\right\}$. Но последнее невозможно, поскольку $S_{1}$ - конечно порожденный $R$-модуль псевдорационального ранга 0 . Из полученного противоречия следует, что $\Delta^{*} M_{X}$ - конечно порожденный $R$-модуль.

Рассмотрим короткую точную последовательность

$$
0 \longrightarrow \Delta^{*} M_{X} \longrightarrow \mathbb{R}^{n} \longrightarrow M / t M \rightarrow 0 .
$$

Так как $\Delta^{*} M_{X}$ конечно порожден, то $M / t M$ конечно представим. Очевидно также, что $M / t M$ - плоский $R$-модуль. А любой плоский конечно представимый модуль является проективным (см., например, [8]).

Отображение $\varphi: \Delta^{*} M_{X} \rightarrow t M$ по закону $\varphi\left(r_{1}, \ldots, r_{n}\right)=r_{1} x_{1}+\cdots+r_{n} x_{n}$, очевидно, является эпиморфизмом. Так как $\operatorname{ker} \varphi=\Delta M_{X}$, то $t M \cong \Delta^{*} M_{X} / \Delta M_{X}$. $R$-модуль $\Delta^{*} M_{X}$ является конечно порожденным; следовательно, $t M$ также конечно порожден. Тогда $t M=\varepsilon_{p_{1}} t M \oplus \cdots \oplus \varepsilon_{p_{s}} t M$, где $\varepsilon_{p_{i}} t M-$ конечно порожденные периодические $\widehat{\mathbb{Z}}_{p}$-модули, которые конечны. Следовательно, $R$-модуль $t M$ является конечным.

Из проективности $R$-модуля $M / t M$ следует, что $M \cong M / t M \oplus t M$. Таким образом, обратное утверждение и теорема доказаны.

Лемма 3. Пусть $L-$ подмодуль конечно порожденного $R$-модуля $M$, для которого $r^{*}(L)=r^{*}(M)$; тогда $L$ конечно порожден.

ДоказатеЛЬСтво. Пусть $M=\left\langle x_{1}, \ldots, x_{n}\right\rangle_{R}, L \subseteq M$ и $r^{*}(M)=r^{*}(L)=k$. И пусть $Y=\left\{y_{1}, \ldots, y_{k}\right\}$ - наибольшее подмножество из $L$, независимое по модулю $T L$, т.е. $Y$ содержит по одному элементу из каждого класса эквивалентности по модулю $T L$, кроме нулевого. Так как $r^{*}(L)=r^{*}(M)$, то $Y$ будет наибольшим независимым по модулю $T M$ множеством в $M$. Тогда

$$
x_{i}=r_{i 1} y_{1}+\cdots+r_{i k} y_{k}+t_{i}, \quad \text { где } \quad r_{i j} \in R, \quad t_{i} \in T M
$$

и $M=\left\langle y_{1}, \ldots, y_{k}, t_{1}, \ldots, t_{n}\right\rangle_{R}$. Так как $t_{1}, \ldots, t_{n} \in T M$, найдется такой идемпотент $\varepsilon \in R$, что $\left\langle t_{1}, \ldots, t_{n}\right\rangle_{R} \subseteq \varepsilon T M$. Рассмотрим следующие разложения:

$$
M=(1-\varepsilon)\left\langle y_{1}, \ldots, y_{k}\right\rangle_{R} \oplus \varepsilon M \quad \text { и } \quad L=(1-\varepsilon) L \oplus \varepsilon L .
$$


Отсюда $(1-\varepsilon) L=(1-\varepsilon)\left\langle y_{1}, \ldots, y_{k}\right\rangle_{R}-$ конечно порожденный $R$-модуль и $\varepsilon L \subseteq \varepsilon M$.

Так как $M$ - конечно порожденный $R$-модуль, то $\varepsilon_{p} M$ - тоже конечно порожденный $R$-модуль, причем структура $R$-модуля на $\varepsilon_{p} M$ совпадает со структурой $\widehat{\mathbb{Z}}_{p}$-модуля. Модуль $\varepsilon_{p} L$ содержится в конечно порожденном $\widehat{\mathbb{Z}}_{p}$-модуле $\varepsilon_{p} M$; следовательно, $\varepsilon_{p} L$ конечно порожден. А так как $\varepsilon L$ раскладывается в прямую сумму модулей вида $\varepsilon_{p} L$, где $p$ пробегает некоторое конечное множество, то $\varepsilon L$ тоже конечно порожден.

Таким образом $R$-модуль $L$ конечно порожденный, как прямая сумма конечно порожденных модулей $(1-\varepsilon) L$ и $\varepsilon L$.

ПрЕДЛОЖЕНИЕ 1. Если у конечно порожденного $R$-модуля существует конечно порожденный модуль псевдорациональных отношений, то все его модули псевдорациональных отношений конечно порожденъ.

ДокАЗАтельство. Рассмотрим $R$-модуль $M$, удовлетворяющий условиям предложения. Пусть $\Delta M_{X}$ - его произвольный модуль псевдорациональных отношений, построенный на системе $X=\left\{x_{1}, \ldots, x_{n}\right\}$.

Как и в доказательстве теоремы 5 построим точную последовательность

$$
0 \longrightarrow \Delta^{*} M_{X} \longrightarrow \mathbb{R}^{n} \longrightarrow M / t M \rightarrow 0 .
$$

Так как $M$ имеет конечно порожденный модуль псевдорациональных отношений, по теореме $5 R$-модуль $M / t M$ проективный. Следовательно, последовательность (10) расщепляется, т.е. $R^{n} \cong \Delta^{*} M_{X} \oplus M / t M$. Здесь $\mathbb{R}^{n}$ и $M / t M$ - конечно порожденные $R$-модули; значит, $\Delta^{*} M_{X}$ - тоже конечно порожденный $R$-модуль.

Учитывая, что $\Delta M_{X} \subseteq \Delta^{*} M_{X}$ и $r^{*}\left(\Delta M_{X}\right)=r^{*}\left(\Delta^{*} M_{X}\right)$, по лемме 3 получаем, что $\Delta M_{X}$ - конечно порожденный $R$-модуль.

СЛЕДСТВИЕ 3. Конечно порожденный $R$-модуль является конечно представленным тогда и только тогда, когда он раскладывается в прямую сумму проективного и конечного $R$-модулей.

СЛЕДСТВИЕ 4. Плоский конечно порожденный модуль над кольцом псевдорациональных чисел является проективным тогда и только тогда, когда он конечно представленный.

Справедливость данных утверждений непосредственно вытекает из теоремы 5.

3. Образующие $R$-модули. Пусть $M$ и $L$ - произвольные $K$-модули. Для них рассмотрим подмодули

$$
S_{L}(M)=\sum_{\varphi: M \rightarrow L} \varphi(M) \quad \text { и } \quad K_{M}(L)=\bigcap_{\varphi: M \rightarrow L} \operatorname{ker} \varphi .
$$

Модуль $S_{L}(M)$ называют следом модуля $M$ в модуле $L$, а фактормодуль $M / K_{M}(L)$ называют коследом модуля $L$ в $M$.

ОПРЕДЕЛЕНиЕ 4. Модуль $M$ над кольцом $K$ называется образующим, если его след в кольце $K$ равен $K$.

Найдем условия, при которых модуль $M$ над кольцом псевдорациональных чисел является образующим.

Так как $S=S_{R}(M)$ - идеал кольца $R$, то из выше сказанного следует, что он однозначно задается множеством $T_{S}=T \cap S$ и своим псевдорациональным рангом. 
ТЕОРема 6. $R$-модуль $M$ является образующим тогда и только тогда, когда выполняются следующие условия:

1) $M$ содержит прямые слагаемые вида $\widehat{\mathbb{Z}}_{p}$ при любом простом $p$;

2) $\operatorname{Hom}_{R}(M, R) \neq \operatorname{Hom}_{R}(M, T)$.

Доказательство. Пусть $S$ - след модуля $M$ в кольце $R$. Равенство $S=R$ выполняется тогда и только тогда, когда $T_{S}=T=\bigoplus_{p \in P} \widehat{\mathbb{Z}}_{p}$ и $r^{*}(S)=1$.

Равенство $\varepsilon_{p} S=\widehat{\mathbb{Z}}_{p}$ равносильно существованию эпиморфизма из $M$ в $\widehat{\mathbb{Z}}_{p}$. Но $\widehat{\mathbb{Z}}_{p}$ - проективный $R$-модуль; следовательно, последнее условие эквивалентно тому, что $M$ имеет прямое слагаемое, изоморфное $\widehat{\mathbb{Z}}_{p}$.

Условие $r^{*}(S)=1$ эквивалентно существованию гомоморфизма $\varphi \in \operatorname{Hom}_{R}(M, R)$ такого, что $\varphi(M) \not \subset T$, т.е. равносильно тому, что

$$
\operatorname{Hom}_{R}(M, R) \neq \operatorname{Hom}_{R}(M, T) .
$$

Таким образом, теорема доказана.

ТЕОрема 7. Модуль $M$ над кольцом псевдорачиональных чисел является образующим тогда и только тогда, когда $M \cong R \oplus X$, где $X$ - некоторый $R$-модуль.

ДокАЗАТЕЛЬСТво. Пусть $M$ - произвольный образующий $R$-модуль. Согласно условию 2 теоремы 6 существует такой гомоморфизм $\varphi$ из $M$ в $R$, что $\varphi(M) \not \subset T$. Тогда идеал $\varphi(M)$ кольца $R$ содержит идемпотент $(1-\varepsilon)$ вида $(\mathrm{b})$, а значит,

$$
\varphi(M)=(1-\varepsilon) R \oplus \varepsilon \varphi(M) .
$$

Композиция эпиморфизма $\varphi: M \rightarrow \varphi(M)$ и проекции $\pi: \varphi(M) \rightarrow(1-\varepsilon) R$ есть эпиморфизм из $M$ в $(1-\varepsilon) R$. Но $(1-\varepsilon) R$ является проективным $R$-модулем; следовательно, $M$ содержит прямое слагаемое вида $(1-\varepsilon) R$, т.е.

$$
M \cong(1-\varepsilon) R \oplus X_{1}
$$

Из условия 1 теоремы 6 следует, что $R$-модуль $M$ содержит также прямое слагаемое, изоморфное модулю $\varepsilon R$, а значит, $M \cong R \oplus X$.

Обратное утверждение очевидно.

Из теорем 6 и 7 вытекают довольно интересные следствия.

СлЕДСТВИЕ 5. Если $M$ - произволъный модуль над колъцом псевдорациональных чисел, то условие $\operatorname{Hom}_{R}(M, R) \neq \operatorname{Hom}_{R}(M, T)$ равносильно тому, что $M$ содержит прямое слагаемое вида $(1-\varepsilon) R$.

СлеДСТвИЕ 6. Пусть $M-$ конечно порожденный $R$-модуль. Если

$$
r^{*}(M)-r^{*} \operatorname{Hom}(M, R) \leqslant 1,
$$

то $M$ раскладывается в прямую сумму циклических $R$-модулей.

ДокАЗАТЕЛЬСТвО. Пусть модуль $M$ удовлетворяет условиям данного утверждения и $r^{*}(M)=n$; тогда $r^{*} \operatorname{Hom}_{R}(M, R) \geqslant n-1$, а значит, по следствию 5 имеет место прямое разложение

$$
M \cong(1-\varepsilon) \mathbb{R}^{n-1} \oplus X,
$$


причем $X$ - конечно порожденный $R$-модуль псевдорационального ранга 1 .

В [9] доказано, что конечно порожденный $R$-модуль псевдорационального ранга 1 раскладывается в прямую сумму циклических $R$-модулей. Учитывая, что $(1-\varepsilon) R-$ также циклический $R$-модуль, получаем справедливость следствия.

Отметим, что в [9] показано, что если конечно порожденный $R$-модуль $X$ имеет псевдорациональный ранг 1 , то $X \cong R_{\chi_{1}} \oplus K_{\chi_{2}} \oplus \cdots \oplus K_{\chi_{n}}$. Таким образом, если модуль $M$ удовлетворяет условиям следствия 6 , то он имеет вид

$$
M \cong(1-\varepsilon) \mathbb{R}^{n-1} \oplus R_{\chi_{1}} \oplus K_{\chi_{2}} \oplus \cdots \oplus K_{\chi_{n}} .
$$

ЛЕмма 4. Если $M$ - конечно порожденный $R$-модуль, то

$$
\operatorname{Hom}_{R}(M, T)=T \cdot \operatorname{Hom}_{R}(M, R) .
$$

ДоказАтельство. Так как $T \cdot \operatorname{Hom}_{R}(M, R) \subseteq \operatorname{Hom}_{R}(M, T)$, достаточно показать, что любой гомоморфизм $\varphi$ из $M$ в $T$ лежит в $T \cdot \operatorname{Hom}_{R}(M, R)$.

Пусть $\varphi \in \operatorname{Hom}_{R}(M, T),\left\{x_{1}, \ldots, x_{n}\right\}$ - система порождающих $R$-модуля $M$. Тогда существует такое $\varepsilon \in R$, что $\varphi\left(x_{i}\right) \subseteq \varepsilon R$ для любого $i \in\{1, \ldots, n\}$. Отсюда следует, что $\varphi(M) \subseteq \varepsilon R$ и $\varphi \in T \cdot \operatorname{Hom}_{R}(M, R)$.

Для произвольного конечно порожденного модуля $M$ над кольцом псевдорациональных чисел найдем строение $R$-модуля $\operatorname{Hom}_{R}(M, R)$.

Модуль $M$ можно представить в виде $M \cong(1-\varepsilon) \mathbb{R}^{n} \oplus L$, где $L$ не содержит прямых слагаемых вида $(1-\varepsilon) R$ ни для какого идемпотента вида (b). Тогда в силу следствия 5

$$
\operatorname{Hom}_{R}(M, R) \cong\left[\bigoplus_{n} \operatorname{Hom}((1-\varepsilon) R, R)\right] \oplus \operatorname{Hom}_{R}(L, T) .
$$

По следствию 4

$$
\operatorname{Hom}_{R}(L, T)=T \cdot \operatorname{Hom}_{R}(L, R) \cong \bigoplus_{p \in P} \operatorname{Hom}\left(\varepsilon_{p} L, \widehat{\mathbb{Z}}_{p}\right) .
$$

Так как $L$ - конечно порожденный $R$-модуль, то $\varepsilon_{p} L-$ конечно порожденный $\widehat{\mathbb{Z}}_{p^{-}}$ модуль и, следовательно,

$$
\varepsilon_{p} L \cong \bigoplus_{m_{p}} \widehat{\mathbb{Z}}_{p} \oplus \mathbb{Z}\left(p^{k_{1 p}}\right) \oplus \cdots \oplus \mathbb{Z}\left(p^{k_{s p}}\right) .
$$

Отсюда

$$
\operatorname{Hom}\left(\varepsilon_{p} L, \widehat{\mathbb{Z}}_{p}\right) \cong \bigoplus_{m_{p}} \widehat{\mathbb{Z}}_{p}
$$

Таким образом, из (11)-(13) следует, что

$$
\operatorname{Hom}_{R}(M, R) \cong(1-\varepsilon) \mathbb{R}^{n} \oplus\left[\bigoplus_{p \in P} \bigoplus_{m_{p}} \widehat{\mathbb{Z}}_{p}\right]
$$

СлЕДСТВИЕ 7. Если $M$ - конечно порожденный $R$-модуль, mо $\operatorname{Hom}_{R}(M, R)$ является проективным $R$-модулем конечного псевдорачионального ранга. 
Если $M$ не конечно порожденный $R$-модуль, то $\operatorname{Hom}_{R}(M, R)$ не обязан являться проективным модулем. Например, если $M=T$, то

$$
\operatorname{Hom}_{R}(T, R)=\operatorname{Hom}_{R}(T, T) \cong \prod_{p \in P} \widehat{\mathbb{Z}}_{p} .
$$

Однако нетрудно видеть, что модуль $\operatorname{Hom}_{R}(M, R)$ всегда является плоским.

Далее, опишем класс $R$-модулей с нулевым коследом кольца $R$.

Теорема 8. $R$-модуль $M$ имеет нулевой кослед кольца $R$ тогда и только тогда, когда $M$ не содержит прямых слагаемых вида $\widehat{\mathbb{Z}}_{p}$ ни при каком простом $р$.

ДокАЗАТЕЛЬство. Пусть $M$ не содержит прямых слагаемых вида $\widehat{\mathbb{Z}}_{p}$. В силу проективности $R$-модуля $\widehat{\mathbb{Z}}_{p}$ это равносильно тому, что не существует эпиморфизма из $M$ в $\widehat{\mathbb{Z}}_{p}$. Так как любой ненулевой подмодуль в $\widehat{\mathbb{Z}}_{p}$ изоморфен $\widehat{\mathbb{Z}}_{p}$, любой гомоморфизм из $M$ в $\widehat{\mathbb{Z}}_{p}$ нулевой.

Таким образом, $\operatorname{Hom}_{R}(M, T)=0$, а значит, и $\operatorname{Hom}_{R}(M, R)=0$, т.е. модуль $M$ имеет нулевой кослед кольца $R$.

Заметим, что в силу результатов А.А. Фомина [1] класс конечно порожденных $R$-модулей с нулевым коследом кольца $R$ это в точности класс $\mathscr{G}$, который ввели С. Глаз и У. Уиклесс в [10].

\section{СПИСОК ЦИТИРОВАННОЙ ЛИТЕРАТУРЫ}

[1] A. A. Fomin, "Some mixed abelian groups as modules over the ring of pseudo-rational numbers", Abelian Groups and Modules, Trends in Mathematics, Birkhäuser-Verlag, Basel, 1999, 87-100.

[2] П. А. Крылов, Е. Г. Пахомова, Е. И. Подберезина, "Об одном классе смешанных абелевых групп", Вестн. Томского ун-та, 269 (2000), 47-51.

[3] A. A. Fomin, "Quotient divisible mixed groups", Contemp. Math., 273 (2001), 117-128.

[4] П. А. Крылов, "Смешанные абелевы группы как модули над своими кольцами эндоморфизмов", Фундамент. и прикл. матем., 6:3 (2000), 793-812.

[5] С.В. Чеглякова, "Инъективные модули над кольцом псевдорациональных чисел", Фундамент. и прикл. матем., 7:2 (2001), 627-629.

[6] А. В. Царев, "Псевдорациональный ранг абелевой группы", Сиб. матем. ж., 46:1 (2005), 312-325.

[7] Л. Фукс, Бесконечные абелевы группы, 1, 2, Мир, М., 1974, 1977.

[8] К. Фейс, Алгебра: кольца, модули и категории, 1, Мир, М., 1977.

$[9]$ А. В. Царев, Конечно порожденные $R$-модули, Науч. труды мат. фак-та МПГУ, Прометей, М., 2000.

[10] S. Glaz, W. Wickless, "Regular and principal projective endomorphism rings of mixed abelian groups", Comm. Algebra, 22 (1994), 1161-1176.

\section{А. В. Царев}

Поступило

Московский педагогический государственный университет

01.11 .2005

E-mail: an-tsarev@yandex.ru, algebra@mpgu.edu.ru 